\title{
Comparison of computed Tomography and ultrasonography in evaluating causes of obstructive jaundice
}

\author{
Kaushal L ${ }^{1}$, Patil A ${ }^{2}$, Ahirwar C.P ${ }^{3}$, Sharma K \\ ${ }^{1}$ Dr. (Mrs.) Lovely Kaushal, Professor \& Head of the Department, ${ }^{2}$ Dr. Abhijit Patil, Associate Professor, ${ }^{3}$ Dr. Chandra \\ Prakash Ahirwar, Assistant Professor, ${ }^{4}$ Dr. Kshitij Sharma, Post graduate student, all authors are attached with \\ Department of Radio diagnosis and Imaging, Gandhi Medical College, Bhopal, Madhya Pradesh, India.
}

Address for Correspondence: Dr. Kshitij Sharma, Email: stormoffire76@gmail.com

\begin{abstract}
Objective: To observe and compare the role of ultrasonography and computed tomography in evaluation of causes of obstructive jaundice. Materials and Methods: A total of 100 patients were enrolled in this cross-sectional study done in Department of Radiodiagnosis and Imaging, Gandhi Medical College, Bhopal. Results: Computed tomography and ultrasonography were able to detect the presence of biliary obstruction in $100 \%$ of cases. Liver function tests were altered in all the patients with alkaline phosphatase raise out of proportion to the AST/ALT. The highest incidence of biliary obstruction was found in 61-70 years' age group with mean $( \pm \mathrm{SD})$ age of the patient was $62.7 \pm 12.64$ years. Sensitivity, specificity and accuracy of computed tomography and ultrasonograhy in detecting the various causes of obstructive jaundice were $90.85 \%, 99.21 \%, 98.15 \%$ and $84.15 \%, 98.86 \%$ and $97 \%$ respectively. Conclusions: Computed tomography has a high sensitivity, specificity and accuracy in detecting the causes of biliary obstruction. Considering these attributes, computed tomography can be used as an effective diagnostic modality in cases of obstructive jaundice. Accuracy and specificity for ultrasonography is high in detecting the causes of biliary obstruction with a slightly low sensitivity. Hence, ultrasonography can be used as an effective screening modality in cases of obstructive jaundice.
\end{abstract}

Keywords: Computed Tomography, Ultrasonography, Obstructive jaundice, Biliary obstruction.

\section{Introduction}

The main goals of any imaging procedure in clinically suspected cases of obstructive jaundice are to confirm the presence of obstruction, its location, extent, probable cause, and to provide a sufficiently accurate overview of the biliary tree that will help the surgeon to determine the approach to each individual case $[1,2]$.

Obstructive jaundice can be caused by a plethora of conditions. These include benign as well as benign and malignant conditions. Obstructive jaundice can be caused by the obstruction of the bile duct as with gall and CBD stones, strictures, malignancy, such as cholangiocarcinoma (in which the jaundice is persistent and progressive), periampullary carcinoma, carcinoma gall bladder and carcinoma head of pancreas, Castlemann disease, Caroli's syndrome and metastatic liver tumor [3].

Manuscript received $4^{\text {th }}$ July 2016

Reviewed: $14^{\text {th }}$ July 2016

Author Corrected: $25^{\text {th }}$ July 2016

Accepted for Publication $8^{\text {th }}$ August 2016
USG is fairly accurate to detect dilated and non-dilated bile ducts. USG allows dynamic and real time evaluation of the biliary tree. Diagnostic procedures using ultrasound are painless, harmless, relatively inexpensive, easily available and free of ionizing radiation [4].

Gross intrahepatic dilatation is easy to detect sonographically and result in the "too many tubes" sign, created by the increased number of radiolucent channels in the liver, or the "parallel channel sign", formed by dilated intrahepatic ducts running anterior and parallel to the portal vein tributaries [5].

The normal diameter of CHD measures 4-5 $\mathrm{mm}$ or less on sonograms. The CBD measures $4-6 \mathrm{~mm}$ normally, with a 6-7 $\mathrm{mm}$ diameter considered equivocal.

A diameter of more than $8 \mathrm{~mm}$ is indicative of ductal dilatation [6]. 
Computed tomography is highly accurate to detect dilated and non-dilated bile ducts. CT offers a comprehensive analysis of liver as well as extrahepatic abdomen and pelvis. The ability to provide multiplanar and 3D reconstructions greatly add to diagnostic accuracy of computed tomographic scans. However, computed tomography is potentially hazardous due to its use of ionizing radiation. The average size of the normal intrahepatic ducts is $2 \mathrm{~mm}$ in the central liver and $1.8 \mathrm{~mm}$ in the periphery. Bile ducts appear as water density tubular branching structures converging at the portahepatis. The left and right hepatic ducts course through portahepatis and join to form the CHD lying anterior to the portal vein [7].

The CHD and CBD are usually visible within the hepato-duodenal ligament. The proximal hepatic duct forms a fairly straight, thin walled, low density tube antero-lateral to the portal vein, angling towards the midline. The distal CBD appears on cross section as a circular, low density structure in the pancreatic head or in a groove posterior to the pancreatic head. The duct wall may be discerned separately with a mean thickness of $1 \mathrm{~mm}$ and maximal thickness of $1.5 \mathrm{~mm}$. contrast enhancement of the duct may occur [7]. The normal CHD on CT is $3-6 \mathrm{~mm}$ in diameter and $8-9 \mathrm{~mm}$ is considered dilated [8].

Our institution has a fair influx of patients suffering from obstructive jaundice with the patients constituting a fair number of hospital admissions. Computed tomography and ultrasonography are the primary modalities used in the evaluation of obstructive jaundice. As these modalities are fairly easily available, and constitute the prima facie radiological investigation for the condition, this study is designed to evaluate the diagnostic role and accuracy of computed tomography and ultrasonography in clinically suspected cases of obstructive jaundice.

\section{Materials and Methods}

This study was carried out in the Department of Radiodiagnosis and Imaging, Gandhi Medical College, Bhopal from January 2014 to December 2015. 100 clinically suspected cases of obstructive jaundice were included in the study by simple random sampling. The presumptive diagnosis was based on combination of clinical and laboratory parameters including itching, weight loss, icterus, upper abdominal mass, raised serum values of liver enzymes with alkaline phosphatase raised out of proportion to AST/ALT.
Prior to the commencement of the study the research protocol was approved by ethical review committee and scientific research committee of Gandhi Medical College, Bhopal.

The aim of this study along with details of procedure, involved risk and benefits were explained to the patients and informed consent was taken. Data collection was done according to the afore-structured preformat.

Scanning protocol- The scan was done after 6 hours fast so that gall bladder is not contracted. An initial survey of gall bladder, biliary tree, liver, pancreas and duodenum was done with the patient mainly in supine and left lateral decubitus positions. Organs were visualized in longitudinal and transverse planes in midline, parasagittal, midclavicular, mid-axillary and intercostal views.

An initial plain CT was obtained and then another post contrast scan was obtained after administration of contrast agent Diatrizoate meglumine and Diatrizoate sodium $76 \%$ both orally and iv in appropriate concentration and dosage. Low density oral contrast material was given prior to the procedure. 1000 to 1500 cc of contrast was given $30 \mathrm{~min}$ prior to the procedure.

The post contrast scanning protocols were according to the organ predominantly involved as practiced in our institute.

The size of intrahepatic and extra hepatic biliary tree, maximum transverse diameter of main pancreatic duct, maximum transverse diameter of common duct, lumen and size of gall bladder, presence of choledocholithiasis or cholelithiasis/ size if present, presence of any mass lesion/ maximum antero-posterior and transverse diameter if present, presence of lymphadenopathy, (periportal, peripancreatic, pre and para aortic, retroperitoneal), presence of narrowing / strictures of biliary tree, presence of focal dilatation of intra and/or extra-hepatic bile ducts, presence of ascites. The findings were correlated with histopathological reports.

Statistical analysis- Statistical analysis was done by computer software devised as the statistical package for social sciences (SPSS). The results were summarized as tables and charts. The sensitivity, specificity, positive predictive value, negative predictive value and accuracy of computed tomography and ultrasonography as diagnostic modalities were calculated. A $p$ value of $<0.05$ was considered significant. 


\section{Results}

Female cases were 55 (55\%) and male were 45 (45\%). The highest incidence of biliary obstruction was found in 61-70 years' age with mean $( \pm \mathrm{SD})$ age of the patient was $62.7 \pm 12.64$ years. The levels of serum alkaline phosphatase, aspartate aminotransferase and alanine aminotransferase were raised with alkaline phosphatase raised out of proportion to the other two.

Figure 1, shows the ultrasonographic features of dilated biliary tree. Figure 2, shows the computed tomography features of dilated biliary tree.

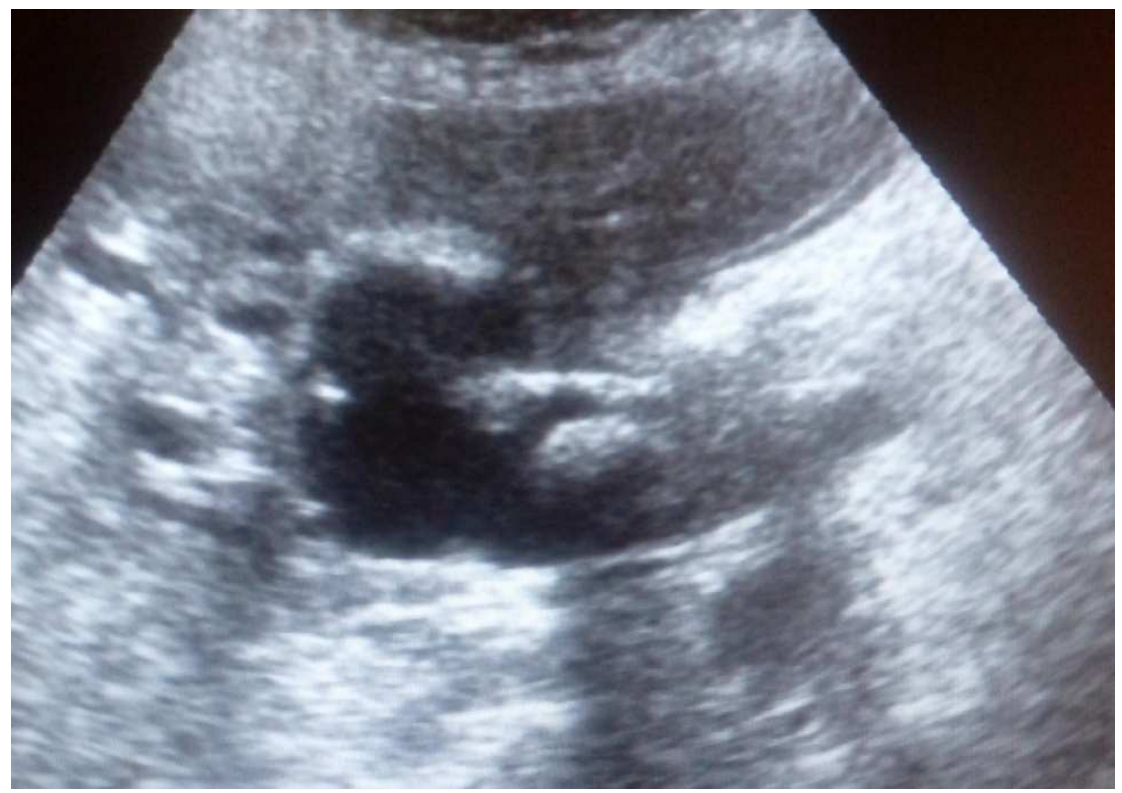

Fig-1: Grossly dilated common duct and intrahepatic biliary radicles due to presence of large obstructing common duct stone.

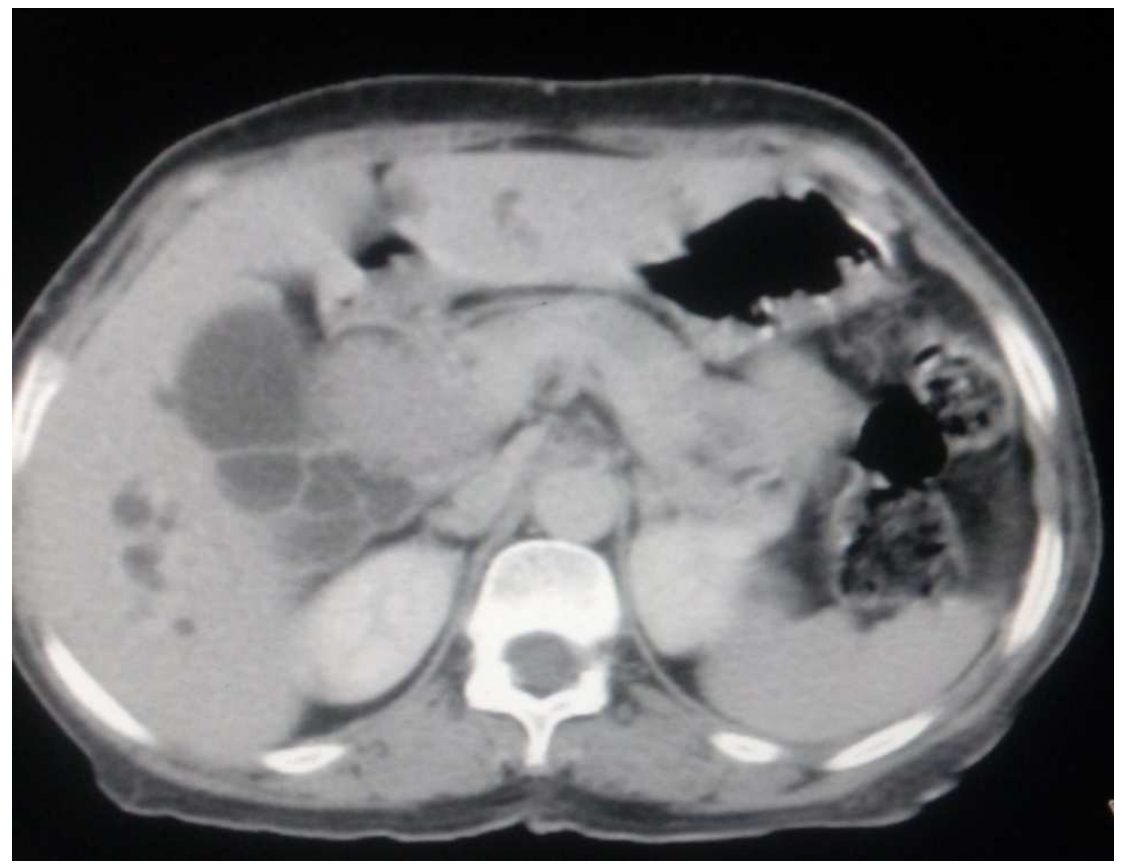

Fig-2: Dilated gall bladder, common duct and intrahepatic biliary ductules due to presence of large mass lesion diagnosed on CT as cholangiocarcinoma. It was further confirmed on histopathology as cholangiocarcinoma. 
As shown in table 1, malignancy was the cause of obstructive jaundice in $80 \%$ of cases as compared to benign etiology in $20 \%$ of cases. Carcinoma of gall bladder was the leading cause of obstructive jaundice.

Table-1: Distribution of cases according to the cause of obstructive jaundice.

\begin{tabular}{|l|c|c|}
\hline Causes of obstructive jaundice & No. of Patients & \% of Patients \\
\hline 1. Carcinoma GB & 41 & 41 \\
\hline 2. Cholangio-Carcinoma & 17 & 17 \\
\hline 3. Pancreatic Head Ca & 11 & 11 \\
\hline 4. Choledocho-lithiasis & 7 & 7 \\
\hline 5. Stricture & 5 & 5 \\
\hline 6. Pancreatitis & 4 & 4 \\
\hline 7. Hepatocellular Carcinoma & 3 & 3 \\
\hline 8. Metastases & 3 & 3 \\
\hline 9. Choledochal Cyst & 3 & 3 \\
\hline 10. Lymphadenopathy Portal & 3 & 1 \\
\hline 11. Pancreatic Metastases & 1 & 1 \\
\hline 12. Carcinoma Duodenum & 1 & 1 \\
\hline 13. Mirizzi syndrome & 1 & 3 \\
\hline
\end{tabular}

Overall, computed tomography and ultrasonography were $100 \%$ sensitive in detecting biliary obstruction. However, the sensitivity decreased for delineating the cause and level of obstruction.

Table-2: Diagnostic value of Ultrasonography in evaluating findings in cases of obstructive jaundice.

\begin{tabular}{|c|c|c|c|c|c|c|c|c|}
\hline ڤ్ & 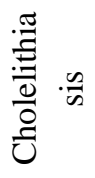 & 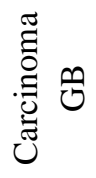 & 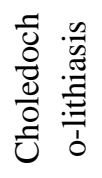 & 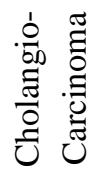 & 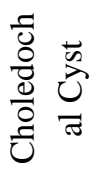 & 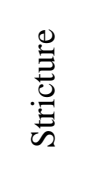 & 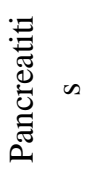 & 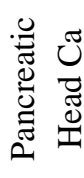 \\
\hline Sensitivity & 94.23 & 82.9 & 68.42 & 82.4 & 100.0 & 100.0 & 100.0 & 81.8 \\
\hline Specificity & 97.92 & 98.3 & 98.77 & 91.6 & 100.0 & 100.0 & 100.0 & 98.9 \\
\hline PPV & 98.0 & 97.1 & 92.86 & 66.7 & 100.0 & 100.0 & 100.0 & 90.0 \\
\hline NPV & 94.0 & 89.2 & 93.02 & 96.2 & 100.0 & 100.0 & 100.0 & 97.8 \\
\hline Accuracy & 96.0 & 92.0 & 93.0 & 90.0 & 100.0 & 100.0 & 100.0 & 97.0 \\
\hline
\end{tabular}

As shown in table 2, sensitivity of ultrasonography was in range of $80-90 \%$ in for most of the causes of obstructive jaundice except for choledocholithiasis in which case it was $68.42 \%$. Positive predictive value of ultrasonography was above $90 \%$ for most diagnosis except for cholangiocarcinoma in which it was low. Diagnostic accuracy of ultasonography was above $90 \%$ for all the findings. Sensitivity of ultrasonography was $94.23 \%$ for cholelithiasis which was a common associated finding seen in the cases of obstructive jaundice.

Table-3: Diagnostic value of computed tomography in evaluating findings in cases of obstructive jaundice.

\begin{tabular}{|c|c|c|c|c|c|c|c|c|c|}
\hline 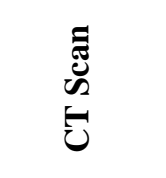 & 胥 & ڤే & 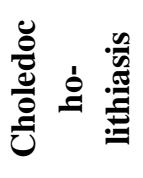 & 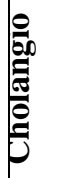 & , & 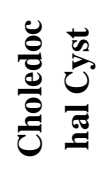 & : & ص & 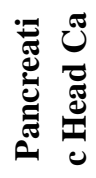 \\
\hline Sensitivity & 78.85 & 90.2 & 84.21 & & 88.2 & 100 & 100 & 100 & 90.9 \\
\hline Specificity & 97.92 & 98.3 & 98.77 & & 95.2 & 100 & 100 & 100 & 98.9 \\
\hline PPV & 97.62 & 97.4 & 94.12 & & 78.9 & 100 & 100 & 100 & 90.9 \\
\hline NPV & 81.03 & 93.5 & 96.39 & & 97.5 & 100 & 100 & 100 & 98.9 \\
\hline Accuracy & 88 & 95 & 96 & & 94 & 100 & 100 & 100 & 98 \\
\hline
\end{tabular}


As shown in table 3, sensitivity of computed tomography was above of $85 \%$ in for all the causes of obstructive jaundice. Positive predictive value of computed tomography was above $90 \%$ for most diagnosis except for cholangiocarcinoma in which it was low. This was due to number of infiltrative large hilar cholangiocarcinomas. Diagnostic accuracy of computed tomography was above $94 \%$ for all the causes. Sensitivity of computed tomography was $78.85 \%$ for cholelithiasis which was a common associated finding seen in the cases of obstructive jaundice.

The present study findings indicate that computed tomography is a more effective diagnostic modality as compared to ultrasonography for most of causes of obstructive jaundice.

\section{Discussion}

In previous studies, the mean age of presentation of biliary obstruction were $48.42 \pm 1.6$ years [4] and 48.14 \pm 12.55 [7] as compared to $62.7 \pm 12.64$ in our study, which was considerably lower. An increased preponderance of involvement of elderly population is seen. The maximum number of patients $45(45 \%)$ were seen in 61-70 years of age group.

No significant difference was seen in prevalence of obstructive jaundice in males and females. However, female preponderance was seen in the cases of carcinoma of gall bladder. This observation may be attributed to the fact that incidence of cholelithiasis was higher in females.

Malignancy was cause of obstructive jaundice in $80 \%$ of cases. Most common as well as most common malignant cause of obstructive jaundice was carcinoma of gall bladder 41/100 (41\%), followed by cholangiocarcinoma $17 / 100(17 \%)$ cases. The most common benign cause was choledocholithiasis causing obstruction in $7 / 100(7 \%)$ of cases, followed by benign biliary strictures (5\%). K. Siddique et al (2007) [10], in their study found that Commonest malignancy was Carcinoma (Ca) of the head of pancreas $(30 \%)$ followed by $\mathrm{Ca}$ gall bladder $(13.33 \%)$ and cholangiocarcinoma (11.66\%). Naffisa Adedin [4] et al. reported carcinoma gall bladder as the most common etiology of obstructive jaundice.

Serum alkaline phosphatase, aspartate aminotransferase and alanine aminotransferase were raised in $100 \%$ of study subjects with serum alkaline phosphatase raised out of proportion to the other two. In obstructive jaundice, serum alkaline phosphatase is usually more than three times the upper limit of normal (40-125 U/1). [11]

In the present study, sensitivity, specificity, PPV, NPV and accuracy of ultrasonography for detection of choledocholithiasis were $68.42 \%, 98.77 \%, 92.86 \%$, $93.02 \%$ and $93 \%$ respectively. Amandeep Singh et al.
(2014) [12] in their study found the diagnostic accuracy, sensitivity and specificity of USG for choledocholithiasis were 96\%, 93.3\% and $97.14 \%$ respectively. Naffisaadedin et al (2012)[4] in their study found that the sensitivity, specificity, accuracy, PPV and NPV of USG for evaluation of choledocholithiasis were $62.5 \%, 100 \%, 94.7 \%, 100 \%, 94.2 \%$ respectively. In another study, ultrasonography correctly identified ductal stones as cause of obstruction in $71 \%$ of cases. [13] Ultrasonography could not detect choledocholithiasis in some cases due to poor visualisation of distal common bile duct owing to bowel gas and obesity.

Sensitivity, specificity, PPV, NPV and accuracy of CT for detection of choledocholithiasis were $84.21 \%$, $98.77 \%, 94.12 \%, 96.39 \%, 96 \%$ respectively. Amandeep Singh et al. (2014) [12] in their study found the diagnostic accuracy, sensitivity and specificity of CT for choledocholithiasis were $94.29 \%, 75 \%$ and $96.77 \%$ respectively. Naffisaadedin et al (2012)[4] in their study found that the the sensitivity, specificity, accuracy, PPV and NPV of CT for evaluation of choledocholithiasis were $75 \%, 100 \%, 96.5 \%, 100 \%, 96.1 \%$ respectively. Stephan W. Anderson et al (2006) [14] found in their study that the overall sensitivity of CT for diagnosis of choledocholithiasis between the two observers ranged from $69 \%$ to $87 \%$, specificity from $83 \%$ to $92 \%$, and accuracy from $84 \%$ to $88 \%$.

$41 \%$ of the cases were diagnosed with carcinoma of gall bladder. Sensitivity, specificity, PPV, NPV and accuracy of USG for detection of carcinoma gall bladder were $82.9 \%, 98.3 \%, 97.1 \%, 89.2 \%, 92 \%$ respectively with a $\mathrm{p}$ value $<0.001$. Naffisaadedin et al (2012) [4] in their study found that the sensitivity, specificity, accuracy, PPV and NPV of USG for evaluation of CA GB were 95\%, 94.6\%, 93\%, 90.5\%, $97.2 \%$ respectively. Khalili and Wilson (2005) [15] in their study estimated the sensitivity of USG in diagnosis of Gall Bladder malignancy to be 94\%. Yeh [16] observed the accuracy of ultrasonography in the 
diagnosis of gall bladder carcinoma to be $84.6 \%$. The present study showed a similar accuracy with a lower sensitivity.

Sensitivity, specificity, PPV, NPV and accuracy of CT for detection of carcinoma gall bladder were $90.2 \%$, $98.3 \%, 97.4 \%, 93.5 \%, 95 \%$ respectively with a p value $<0.001$. Naffisaadedin et al $(2012)^{4}$ in their study found that the sensitivity, specificity, accuracy, PPV and NPV of CT for evaluation of CA GB were $100 \%$, $100 \%, 100 \%, 100 \%, 100 \%$ respectively. Ghafoor N. et al (2006) [17] in their study observed $93.3 \%$ sensitivity of computed tomography for evaluation of gall bladder malignancy. Kumran et al (2002) [18] found the accuracy of CT in the diagnosis of GB mass to be 93.3\%. Yoshimitsu et al (2002) [19] in their study found that the sensitivity and accuracy of CT for detection of Gall bladder mass was $80 \%$ and $86 \%$ respectively. Sensitivity of computed tomography in different studies is comparable.

Loss of fat planes with infiltration into the liver parenchyma was present in $31 / 41(75.6 \%)$ cases. S. Pradhan et al (2002) [20] in their study found presence of liver infiltration in $74 \%$ of cases of carcinoma of gall bladder.

Sensitivity, specificity, PPV, NPV and accuracy of USG for detection of cholangiocarcinoma were $82.4 \%$, $91.6 \%, 66.7 \%, 96.2 \%, 90 \%$ respectively with a p value $<0.001$. Amandeep Singh et al. [12] (2014) in their study found the diagnostic accuracy, sensitivity, specificity and NPV of USG for cholangiocarcinoma was $96 \%, 66.67 \%, 100 \%, 95.65 \%$ respectively. L E Hann et al (1997) [21] found in their study that ductal masses were revealed by sonography in $87 \%$.

Sensitivity, specificity, PPV, NPV and accuracy of CT for detection of cholangiocarcinoma were $88.2 \%$, $95.2 \%, 78.9 \%, 97.5 \%, 94 \%$ respectively with a p value $<0.001$. Amandeep Singh et al. [12] (2014) in their study found the diagnostic accuracy, sensitivity and specificity of CT for cholangiocarcinoma was $97.14 \%, 83.33 \%$ and $100 \%$ respectively.

$11 / 17(64.7 \%)$ cases of cholangiocarcinoma were extrahepatic, 5/17 (29.4\%) cases were hilar, 1/17 (5.9\%) cases were intrahepatic variety.

Sensitivity, specificity, PPV, NPV and accuracy of USG for detection of carcinoma head of pancreas were $81.80 \%, 98.9 \%, 90 \%, 97.8 \%, 97 \%$ respectively with a $p$ value $<0.001$. Naffisa adedin et al (2012) [4] in their study found that the sensitivity, specificity, accuracy, PPV and NPV of USG for evaluation of CA Pancreas were $80.0 \%, 97.6 \%, 93 \%, 92.3 \%, 93.2 \%$ respectively. Thomas MJ et al (1982) [22] in their study found that USG was $97 \%$ sensitive with $100 \%$ PPV, accuracy of USG was $80.0 \%$. Hessel et al (1982) [23] found that USG has a sensitivity of $69 \%$ and specificity of $82 \%$.

Sensitivity, specificity, PPV, NPV and accuracy of CT for detection of carcinoma of pancreas were $90.90 \%$, $98.9 \%, 90.9 \%, 98.9 \%$, 98\% respectively with a p value $<0.001$. Naffisaadedin et al (2012)[4] in their study found that the sensitivity, specificity, accuracy, PPV and NPV of CT for evaluation of CA Pancreas were $93.3 \%, 97.6 \%, 96.5 \%, 93.3 \%, 97.6 \%$ respectively.

Thomas MJ et al (1982) [22] in their study found that accuracy of CT was 93\%. Hessel et al (1982) [23] found that that CT has a sensitivity of $87 \%$ and specificity of $90 \%$.

Sensitivity, specificity, PPV, NPV and accuracy of USG for detection of cholelithiasis were $94.23 \%$, $97.92 \%, 98 \%, 94 \%, 96 \%$ respectively. Weltman DI et al (1994)[24] reported the accuracy of USG for detection of cholelithiasis to be $94 \%$. Sensitivity, specificity, PPV, NPV and accuracy of CT for detection of cholelithiasis were $78.85 \%, 97.92 \%, 97.62 \%, 81.03 \%$, $88 \%$ respectively. Paulson EK et al (2000) [25] reported the sensitivity of CT for detection of gall stones to be $75 \%$.

Sensitivity, specific, PPV, NPV and accuracy of USG for detection of metastases were $88.90 \%$, 97.8\%, $80.0 \%, 98.9 \%, 97 \%$ respectively with a $\mathrm{p}$ value $<0.001$. Sensitivity, specific, PPV, NPV and accuracy of CT for detection of metastases were $88.90 \%, 98.9 \%, 88.9 \%$, $98.9 \%, 98 \%$ respectively with a $\mathrm{p}$ value $<0.001$.

Sensitivity, specific, PPV, NPV and accuracy of computed tomography and ultrasonography both for detection of choledochal cyst, biliary stricture, hepatocellular carcinoma and pancreatitis as a cause of obstructive jaundice were 100\%, 100\%, 100\%, 100\%, $100 \%$ respectively. All the cases were Modified Todani Type I choledochal cyst. However, not much about the statistical significance can be said due to the limited number of cases.

The overall sensitivity, specificity, PPV, NPV, accuracy of USG for detecting various causes of obstruction was 
$84.15 \%, 98.86 \%, 91.39 \%, 97.74 \%$ and $97 \%$ respectively with a $\mathrm{p}$ value of $<0.0001$. Satish $\mathrm{K}$. Bhargava et al (2013) [26], in their study found that USG could pick up the presence of biliary obstruction in almost all cases $(100 \%)$. Accurate detection of the level was possible in $98 \%$ of cases and to a much lesser extent the cause of obstruction in $75 \%$ of cases. Naffisaadedin et al (2012) [4] in their study found that sensitivity, accuracy and PPV for USG to detect the cause of biliary obstruction were $68.4 \%, 68.4 \%$ and $100 \%$ respectively.

The overall sensitivity, specificity, PPV, NPV, accuracy of CT for detecting various causes of obstruction was $90.85 \%, 99.21 \%, 94.3 \%, 98.69 \%$ and $98.15 \%$ respectively with a $\mathrm{p}$ value of $<0.0001$. Satish K. Bhargava et al (2013) [26], in their study found that CECT could detect the presence and level of obstruction in all cases (100\%).

Naffisaadedin et al (2012) [4] in their study found that sensitivity, accuracy and PPV for CT to detect the cause of biliary obstruction were $96.5 \%, 96.5 \%$ and $100 \%$ respectively.

\section{Conclusions}

Malignancy was the cause of obstructive jaundice in $80 \%$ of cases as compared to benign causes in $20 \%$ of cases. The most common cause of obstructive jaundice was carcinoma of gall bladder. The most common benign cause of obstructive jaundice was choledocholithiasis. The most common associated finding seen in cases of obstructive jaundice was cholelithiasis followed by lymphadenopathy.

Overall, CT was effective diagnostic modality for all the causes, however, it had a decreased sensitivity for detection of cholelithiasis. With the above statistical evaluation and in accordance with the findings of previous studies it can be safely said that computed tomography is a better diagnostic modality as compared to ultrasonography in clinically suspected cases of obstructive jaundice and provides good quality diagnostic information.

Malignancy with gall bladder carcinoma was found as leading the cause of obstructive jaundice. Further studies investigating into the various predisposing factors for the increased prevalence of GB carcinoma are required.Further studies are required to validate the significance of CT and USG as staging modality in malignant causes of obstructive jaundice.
Funding: Nil, Conflict of interest: None initiated, Permission from IRB: Yes

\section{References}

1. Nadkarni KM, Jahagirdar RR, Kagzi RS, Pinto AC, Bhalerao RA. Surgical obstructive jaundice. J Postgrad Med. 1981 Jan;27(1):33-9.

2. Baron RL, Stanley RJ, Lee JKT, Koehler RE, Melson GL, Balfe DM, Weyman PJ. A prospective comparison of the evaluation of biliary obstruction using computed tomography and ultrasonography. Radiology 1982 Oct; 145(1);91-98.DOI:10.1148/radiology.145.1.7122903.

3. Sharma MP, Ahuja V. Aetiological spectrum of obstructive jaundice and diagnostic ability of ultrasonography: a clinician's perspective. Trop Gastroenterol. 1999 Oct-Dec;20(4):167-9.

4. Naffisaadedin et al, comparison of ultrasonography and computed tomography to evaluate causes of biliary obstruction; Euroasian journal of hepatogastroenterology, 2012July-December;2(2):98-103.

5. Laing FC: The gallbladder and bile ducts. In Rumack CM, Wilson SR, Charboneau JW (Eds): Diagnostic ultrasound ( $2^{\text {nd }}$ ed.)St.Louis, CV Mosby,1998,pp175224.

6. Reinus WR, Shady K, Lind M, Scott R. Ultrasound evaluation of the common duct in symptomatic and asymptomatic patients. Am J Gastroenterol. 1992 Apr; 87 (4):489-92.

7. Baron RL, Tublin ME, Peterson MS. Imaging the spectrum of biliary tract disease. Radiol Clin North Am. 2002 Dec;40(6):1325-54.

8. Co CS, Shea WJ Jr, Goldberg HI. Evaluation of common bile duct diameter using high resolution computed tomography. J Comput Assist Tomogr. 1986 May-Jun;10(3):424-7.

9. Upadhyaya V, Upadhyaya DN, Ansari MA, Shukla VK. Comparative assessment of imaging modalities in biliary obstruction. Indian J Radiol Imaging 2006; 16:577-82.

10. Siddique K, Ali Q, Mirza S, Jamil A, Ehsan A, Latif S, Malik AZ. Evaluation of the aetiological spectrum of obstructive jaundice. J Ayub Med Coll Abbottabad. 2008 Oct-Dec;20(4):62-6. 
11. Britton J, Bickerstaff KI, Savage A. Diseases of biliary tract. In: Morris PJ, Wood WC(Eds). Oxford Textbook of Surgery, ( $2^{\text {nd }}$ edition), Oxford University Press 2000;1685.

12. Singh A, Mann HS, Thukral CL, Singh NR. Diagnostic Accuracy of MRCP as Compared to Ultrasound/CT in Patients with Obstructive Jaundice. J Clin Diagn Res. 2014 Mar;8(3):103-7. doi: 10.7860/ JCDR/2014/8149.4120. Epub 2014 Mar 15.

13. Rigauts H, Marchal G, Van Steenbergen W, Ponette E. Comparison of ultrasound and E.R.C.P. in the detection of the cause of obstructive biliary disease. Rofo. 1992 Mar;156(3):252-7.

14. Anderson SW, Lucey BC, Varghese JC, Soto JA. Accuracy of MDCT in the diagnosis of choledocholithiasis. AJR Am J Roentgenol. 2006 Jul; 187(1):174-80.

15. Khalili K, Wilson SR.The biliary tree and Gall bladder. In: Rumack CM, Wilson SR, Charboneau JW(Eds). Diagnostic Ultrasound $\left(3^{\text {rd }}\right.$ ed $)$, Elsevier, Mosby 2005;142:725-28.

16.Yeh HC. Ultrasonography and computed tomography of carcinoma of the gallbladder. Radiology. 1979 Oct;133(1):167-73.

17. Ghafoor N. Role of ultrasound and computed tomography in the diagnosis of gall bladder malignancy. MD thesis, BIRDEM, Dhaka, Bangladesh, 2006.

18. Kumaran V, Gulati S, Paul B, Pande K, Sahni P, Chattopadhyay K. The role of dual-phase helical CT in assessing resectability of carcinoma of the gallbladder. Eur Radiol. 2002 Aug;12(8):1993-9. Epub 2002 Apr 19.
19. Yoshimitsu K, Honda H, Shinozaki K, Aibe H, Kuroiwa T, Irie H, Chijiiwa K, Asayama Y, Masuda K. Helical CT of the local spread of carcinoma of the gallbladder: evaluation according to the TNM system in patients who underwent surgical resection. AJR Am J Roentgenol. 2002 Aug;179(2):423-8.

20. Pradhan S, Shukla VK, Agrawal S, Dixit VK, Sharma OP. Sonographic and colour doppler morphology in carcinoma gallbladder. Indian J Cancer. 2002 Oct-Dec;39(4):143-8.

21. Hann LE, Greatrex KV, Bach AM, Fong Y, Blumgart LH. Cholangiocarcinoma at the hepatic hilus: sonographic findings. AJR Am J Roentgenol. 1997 Apr; 168(4):985-9.

22. Thomas MJ, Pellegrini CA, Way LW. Usefulness of diagnostic tests for biliary obstruction. Am J Surg. 1982 Jul;144(1):102-8.

23. Hessel SJ. Siegelman SS, McNeil BJ, Sanders R, Adams DF, Alderson PO, Finberg HJ, Abrams HL. A prospective study of evaluation of computed tomography and ultrasound of the pancreas. Radiol 1982 Apr:143(1):129-33.

24. Weltman DI, Zeman RK. Acute diseases of the gallbladder and biliary ducts. Radiol Clin North Am. 1994 Sep;32(5):933-50.

25. Paulson EK. Acute cholecystitis: CT findings. Semin Ultrasound CT MR. 2000 Feb;21(1):56-63.

26. Bhargava SK, Usha $\mathrm{T}$, Bhatt $\mathrm{S}$, Bhargava $\mathrm{S}$. Imaging in obstructive jaundice: A review with our experience JIMSA. 2013;26:12-4.

\section{How to cite this article?}

Kaushal L, Patil A, Ahirwar C.P, Sharma K. Comparison of computed Tomography and ultrasonography in evaluating causes of obstructive jaundice. Int J Med Res Rev 2016;4(8):1448-1455.doi:10.17511/ijmrr.2016.i08.28. 